\title{
Hyperfine-interaction-driven Aharonov-Bohm effect in mesoscopic rings
}

\author{
I. D. Vagner ${ }^{1}$, A. S. Rozhavsky ${ }^{2}$, and P. Wyder $^{1}$ \\ ${ }^{1}$ Grenoble High Magnetic Field Laboratory Max-Planck-Institut für Festkörperforschung and Centre \\ Nationale de la Recherche Scientifique, BP 166, 38042 Grenoble Cedex 09, France \\ E-mail: vagner@labs. polycurs.-gre.fr
}

${ }^{2}$ B. I. Verkin Institute for Low Temperature Physics and Engineering, National Academy of Sciences of Ukraine, 47, Lenin Ave., 310164, Kharkov, Ukraine

E-mali: rozhavsky@ilt.kharkov.ua

Submitted April 21, 1997, revised July 23, 1997

\begin{abstract}
It is shown qualitatively that lifting of the electron spin degeneracy by a hyperfine field, which is generated by a nonequilibrium nuclear spin distribution, and breaking of the left-right symmetry by the spin-orbit interaction in a closed ring produces under certain conditions a persistent current, which demonstrates the Aharonov-Bohm-like oscillations with time in GaAs/AlGaAs-based mesoscopic rings even in the absence of an external magnetic field. The typical time interval of these (meso-nucleospinic) oscillations is of the order of several seconds, which is typical of the nuclear spin relaxation times in heterojunctions.
\end{abstract}

PACS: 31.30.Gs, 72.15.Rn, 73.23.Ps

The persistent currents (PC) in multiconnected mesoscopic conductors reflect the broken clockwiseanticlockwise symmetry, which is usually caused by the external vector potential. Experimentally, PCs are observed when an adiabatically slow, timedependent, external magnetic field is applied along the ring axis [1-3]. The magnetic field variation results in the oscillatory behavior, with the magnetic flux quantum $\Phi_{0}=h c / e$ (or its harmonics), of the diamagnetic moment (the PC), which is a manifestation of the Aharonov-Bohm effect [4].

We propose that the persistent current exists in a GaAs/AlGaAs quantum ring with a nonequilibrium nuclear spin population, even in the absence of an external magnetic field. We predict the ABE-like oscillations of PC with time during the time interval of the order of nuclear spin relaxation time $T_{1}$, which is known to be long in semiconductors at low temperatures. The physics behind these oscillations can be understood along the following lines. The hyperfine field, which is caused by the nonequilibrium nuclear spin population [5], breaks the spin symmetry of conduction electrons which, combined with a strong spin-orbit coupling, results under certain conditions in the breaking of the rotational symmetry of diamagnetic currents in a ring. Under the topologically nontrivial spin distribution, the hyperfine field produces the Berry phase analogous to one which emerges in textured mesoscopic rings [7-11].

We note that this is the first of a series of «meso-nucleospinic» effects, which take place in different systems with broken symmetry due to the combined action of the hyperfine field and spin-orbit coupling.

Let us first examine the nature of the hyperfine electron-nuclear spin interaction and of the electron spin-orbit interaction in typical heterojunctions.

The hyperfine interactions in GaAs heterojunctions and similar quantum Hall systems attracted recently sharply growing theoretical [12] and experimental [13-15] attention. The main physical interest in this subject is based on the fact that the discrete nature of the electron spectrum in these systems results in the exponential $(\exp (\Delta / T)$, where $\Delta$ is the mean electron energy spacing, and $T$ is the temperature dependence of the nuclear spin relaxation times $T_{1}$ on the system parameters). We assume here that similar law should apply for the nanostructures with well-defined size quantization of the electron spectrum. Note that in this case $T_{1}$ is very sensitive to the potential fluctuations caused 
by the inhomogeneous distribution of impurities in a heterojunction. Indeed, as it was discussed in Ref. 16, the impurity potential modulates the spacing, thus providing faster nuclear relaxation.

Intensive experimental studies of this phenomenon have provided a more detailed knowledge of the hyperfine interaction between the nuclear and electron spins in heterojunctions and quantum wells. It was observed that the nuclear spin relaxation time in $\mathrm{GaAs} / \mathrm{AlGaAs}$ is rather long (up to $10^{3} \mathrm{sec}$ ) [13] and the hyperfine field acting on electron spins in this material is extremely high, up to $10^{4} \mathrm{~g}[14,15]$. Based on this knowledge we suggest that once the nonequilibrium population of nuclear spins is created, the hyperfine field may strongly influence the electron transport and persistent currents in nanostructures in sufficiently long time.

In this brief note we show qualitatively that the combined action of the hyperfine nuclear field and the electron spin-orbit interaction breaks the time reversal symmetry in a mesoscopic ring (i.e., a sufficiently small ring so that quantum-mechanical phase coherence is preserved), formed in a GaAs/AlGaAs 2DEG, which results in an oscillating persistent current at millikelvin temperatures. The detailed theory will be published elsewhere.

The contact hyperfine interaction between the electron and nuclear spins is described by the following term in the Hamiltonian [6]:

$$
\hat{H}_{c h f}^{e n}=\frac{8 \pi}{3} \mu_{B} \gamma_{h} \hbar^{2} \sum_{i} I_{i} S \delta\left(r_{e}-R_{i}\right)
$$

where $\mu_{B}$ and $\gamma_{h}$ are the conduction electron and the nuclear magnetons; $I, S, R_{i}$, and $r_{e}$ are the nuclear and the electron spins and position vectors, respectively; and $\delta\left(r_{e}-R_{i}\right)$ reflects the fact that the $s$-electrons have a nonvanishing probability on the nuclei. Since the conduction quasiparticle wavelength in GaAs is much larger than the internuclei distance, it follows from Eq. (1), that once the nuclear spins are polarized, i.e., if $\left\langle\sum_{i} I_{i}\right\rangle \neq 0$ (Refs. 13-15), the electron spins will experience an effective hyperfine field, which lifts the electron spin degeneracy even in the absence of an external magnetic field. In GaAs / AlGaAs one may achieve the spin splitting due to hyperfine field of the order of the Fermi energy: $\mu_{B} H_{c h f} \cong \varepsilon_{F}$ (Refs. 14 and $15)$, which is of the order of $1 \mathrm{~K}$.

Let us assume, therefore, that only one electron spin orientation is populated during the time interval of the order of the nuclear spin relaxation time $T_{1}$. It is quite obvious that under the topologically nontrivial spin texture (see below) switching on electron spin-orbit interaction in the system of spinpolarized electrons will produce a persistent current.

The standard expression for the spin-orbital interaction

$$
\hat{H}_{s o}=\frac{\hbar}{4 m^{2} c^{2}}[\nabla V \times \mathbf{p}]
$$

can be rewritten in the form: $\hat{H}_{\text {so }}=\mathbf{p} \hat{\mathbf{A}}_{\text {eff }}$, where the effective vector potential operator reflects the combined action of the hyperfine field and of the spin-orbit interaction:

$$
\hat{\mathbf{A}}_{\mathrm{eff}}(t)=\frac{\hbar}{4 m c^{2}}[\sigma(t) \times \nabla V]=\hat{\mathbf{A}}_{\mathrm{eff}}^{0} \exp \left(-\frac{t}{T_{1}}\right) .
$$

In what follows we restrict the discussion to the case of GaAs / AlGaAs heterostructure-based mesoscopic rings. Spin-orbit interaction in semiconductors can be caused by impurities, boundaries, etc. In GaAs there is a well-known mechanism, attributable to the crystal anisotropy [17], which can be presented as

$$
\hat{H}_{s o}=\frac{\alpha}{\hbar} \sum_{i}\left[\sigma_{i} \times \mathbf{p}\right] \mathbf{v} .
$$

Here $\alpha=0.6 \cdot 10^{-9} \mathrm{eV} \cdot \mathrm{cm}$ for holes with $m^{*}=0.5 m_{0}$ ( $m_{0}$ is the free electron mass); $\boldsymbol{\sigma}$ and $\mathbf{p}$ are the charge carrier spin and momentum; and $v$ is the normal to the surface.

We obtain for the effective vector potential operator [Eq. (3)] the estimate

$$
\hat{A}_{\mathrm{eff}}^{\mathrm{GaAs}} \cong \frac{\alpha m^{*}}{\hbar} .
$$

Under real conditions we have $\mu_{B} H_{e h f}>>\alpha / L$, where $L$ is the loop perimeter, and the electron spin projection is a well-defined quantum number. The vector potential [Eq. (5)] generates a persistent current, which is the sum over the partial current carried by the individual energy levels with different projections of the electron spin.

The result of summation is an oscillatory dependence of PC on the effective flux across the loop:

$$
2 \pi \varphi=\frac{1}{\hbar} \oint d l\left\langle A_{\mathrm{eff}}^{\mathrm{GaAs}}\right\rangle=\frac{L\langle\langle\alpha\rangle\rangle}{\hbar^{2}}\langle\sigma(t)\rangle
$$

where the brackets stand for quantum averaging, and the double brackets denote

$$
\langle\langle\alpha\rangle\rangle=\frac{1}{L} \oint \alpha(t) d l .
$$


For a micron ring formed in a $\mathrm{GaAs} / \mathrm{AlGaAs}$ $2 \mathrm{DEG}, 2 \pi \varphi \sim 30\langle\sigma(t)\rangle$. The value of $\langle\sigma(t)\rangle$ depends on the particular features of the relaxation dynamics.

To estimate the amplitude of a PC generated by a time-dependent flux [Eq. (6)], we must establish the hierarchy of time scales in a micron ring at the level of millikelvins.

The time scales are the nuclear magnetization relaxation time $T_{1}$, the ballistic time $\hbar / \Delta \propto L / V_{F}$, the phase-breaking time $\tau_{\varphi} \cong \tau_{\text {in }}$, where $\tau_{\text {in }}$ is the time of inelastic electron scattaring, and $\tau_{e}$ is one of the elastic processes.

The time $\tau_{e}$ in quasi-one-dimensional GaAs / $\mathrm{AlGaAs}$ rings is governed mainly by smooth tails of the impurity potential located in the «spacer» $[20,21]$. In an actual experiment [3] the elastic mean free path is of the order of $10 \mu \mathrm{m}$.

The inelastic time $\tau_{\text {in }}$ in quasi-one-dimensional quantum rings is defined by a combined action of the electron-phonon scattering and the elastic scattering [20]. Under the condition $\mu_{B} H_{e h f}$ $\sim \varepsilon_{F}>>\Delta>>T$ the electron-spin-lattice [22] scattering in a ring is suppressed. It is estimated [20] to be $\tau_{\varphi} \cong 10^{-7}-10^{-8}$ sec.

In our case the time $T_{1}$ far exceeds all the microscopic electron times, $T_{1}>>\left(\hbar / \Delta, \tau_{\varphi}, \tau_{e}\right)$, which evidently means that energy relaxation restores the equilibrium distribution of the electron levels, and we have a thermodynamic situation [20]. When $L \sim l_{e} \ll L_{\varphi}$, the persistent current can be calculated as [4]

$$
j_{P C}=-\left.c \frac{\partial F}{\partial \Phi_{\mathrm{ext}}}\right|_{\Phi_{\mathrm{ext}}=0}
$$

where $F$ is the free energy, and $\Phi_{\text {ext }}$ is the probe external flux. The thermodynamic value of $\langle\sigma(t)\rangle$ in Eq. (6) is $\mu_{B} H_{e h f}(t) / \varepsilon_{F} \cong 1$.

In light of the above discussion, we estimate the persistent current in a fully spin-polarized gas when $\Delta>T$ to be

$$
j_{P C} \cong \frac{e V_{F}}{L} \sum_{k=1}^{\infty} \frac{\sin \left(2 \pi k \varphi_{0} \exp \left(-t / T_{1}\right)\right)}{k},
$$

where $\varphi_{0} \cong L\langle\langle\alpha\rangle\rangle \hbar^{-2} m^{*}$.

The oscillations of persistent current arise due to the exponential time dependence of the phase $\varphi_{0} \exp \left(-t / T_{1}\right)$ in Eq. (9), with the time constant $T_{1}$, which in GaAs can be of the order of several seconds at a temperature of several millikelvins [5]. We note the marked difference between the time dependence of standard $\mathrm{AB}$ oscillations, which are usually observed under the condition of linear time variation of the applied magnetic field, and the hyperfine driven oscillations, which die out due to the exponential time dependence of the nuclear polarization.

Let us now consider experimental feasibility of the proposed effect. A nuclear spin configuration should be created when the circulation of the effective vector potential is a nonzero topological invariant [Eq. (6)]. In the geometry where the vector $v$ in Eq. (4) is normal to the heterojunction, either the nuclei, which are pumped along some direction in the plane of the ring, or the spin-orbit coupling should be inhomogeneous along the perimeter, say, due to the inhomogeneous distribution of impurities. Since in a GaAs/AlGaAs heterojunction the characteristic length of the impurity potential is of the order of several hundreds $\AA$, i.e., comparable to the ring width, the mesoscopic sensitivity to a single impurity position may produce a nonvanishing phase [Eq. (6)]. The influence of the long range impurities on the nuclear spin-relaxation time in heterojunctions was studied in Ref. 16. Note that the spin-orbit-produced PC considered above is not averaged in a single ring, in marked contrast with the situation considered in Refs. 8 and 9 .

Other mechanisms connected with external potentials like boundaries, heavy-atom impurities along the ring perimeter, and other imperfections, which may enhance the spin-orbit interaction in these systems, will be considered in detail elsewhere.

In summary we propose here the first in a family of hyperfine-field-driven mesoscopic (mesospinic) orbital effects: the Aharonov-Bohm-like oscillations of a persistent current in a GaAs/AlGaAs mesoscopic ring in the absence of external magnetic field. We note that the large (of the order of $1 \mathrm{~T}$ ) hyperfine field acts on the electron spins only, which results in the nonequilibrium electron spin population. The later, at millikelvin temperatures, under the conditions of the topologically nontrivial texture, $\langle\langle\alpha\rangle\rangle \neq 0$, via the spin-orbit coupling, results in weak persistent currents, which decrease with time due to the nuclear depolarization, during the time interval of the order of the nuclear spin-relaxation time $T_{1}$ which can be sufficiently long at low temperatures.

We acknowledge illuminative discussions with Yu. Bychkov, A. Dyugaev, I. Krive, L. Levy, G. Platero, and A. Zyuzin.

I. V. and P. W . acknowledge the support by GIFGerman Israeli Foundation for Scientific Research and Development, Grant No. 1-0222-136.07/91. 
1. L. P. Levy, G. Dolan, J. Dunsmuir, and H. Bouchiat, Phys. Rev. Lett. 64, 2074 (1990).

2. V. Chandrasekhar et al., Phys. Rev. Lett. 67, 3578 (1991).

3. D. Mailly, C. Chapelier, and A. Benoit, Phys. Rev. Lett. 70, 2020 (1991); Physica B197, 514 (1994).

4. See for a review: I. V. Krive and A. S. Rozhavsky, Int. J. Mod. Phys. B6, 1255 (1992); A. A. Zvyagin and I. V. Krive, Fiz. Nizk. Temp. 21, 688 (1995) [Low Temp. Phys. 21, 533 (1995)].

5. M. I. Dyakonov and V. I. Perel, in: Modern Problems in Condensed Matter Sciences, F. Meier and B. P. Zakharchenya (eds.), North-Holand, Vol. 8 (1984), p. 11.

6. C. P. Slichter, Principles of Magnetic Resonance, Springer Verlag (1991), Second ed.

7. D. Loss, P. Goldbart, and A. V. Balatsky, Phys. Rev. Lett. 65, 1655 (1990).

8. E. N. Bogachek, I. V. Krive, I. O. Kulik, and A. S. Rozhavsky, Mod. Phys. Lett. B5, 1607 (1991).

9. A. G. Aronov and Y. B. Lyanda-Geller, Phys. Rev. Lett. 70, 343 (1993).

10. A. V. Balatsky and B. L. Altshuler, Phys. Rev. Lett. 70, 1678 (1993)

11. T.-Z. Qian and Z.-B. Su, Phys. Rev. Lett. 72, 2311 (1994).

12. I. D. Vagner and T. Maniv, Phys. Rev. Lett. 61, 1400 (1988); see for a recent review I. D. Vagner, Yu. A. By- chkov, A. M. Dyugaev, and T. Maniv, Physica Scripta 66, 158 (1996).

13. A. Berg, M. Dobers, R. R. Gerhardts, and K. von Klitzing, Phys. Rev. Lett. 64, 2563 (1990).

14. K. Wald, L. P. Kouwenhoven, P. L. McEuen, N. C. Van der Vaart, and C. T. Foxon, Phys. Rev. Lett. 73, 1011 (1994).

15. S. E. Barret et al., Phys. Rev. Lett. 75, 4290 (1995); see for a review R. Tycko et al., Science 268, 1460 (1995).

16. S. V. Iordanskii, S. V. Meshkov, and I. D. Vagner, Phys. Rev. B44, 6554 (1991).

17. Yu. A. Bychkov and E. I. Rashba, JETP Lett. 39, 78 (1984).

18. Y. Meir, Y. Gefen, and O. Entin-Wohlman, Phys. Rev. Lett. 63, 798 (1989).

19. O. Entin-Wohman, Y. Gefen, Y. Meir, and Y. Oreg, Phys. Rev. B45, 11890 (1992).

20. E. N. Bogachek, Yu. M. Galperin, M. Jonson, R. I. Shekhter, and T. Swahn, J. Phys.: Condens. Matter 8, 2603 (1996).

21. A. S. Rozhavsky, J. Phys, Condens, Matter 9, 1521 (1997).

22. R. J. Elliott, Phys. Rev. 96, 266 (1954). 